\section{Determinantes do baixo peso ao nascer a partir das Declarações de Nascidos Vivos}

\section{Determinants for low birth weight according to Live Born Certificates}

Emília de Faria Carniel

Maria de Lurdes Zanolli

Maria Ângela Reis de Góes Monteiro Antônio

André Moreno Morcillo

Departamento de Pediatria da Faculdade de Ciências Médicas da UNICAMP

Correspondência: Emília de Faria Carniel, Rua Serra Formosa $n^{\circ}$ 66, Jardim Paranapanema, Campinas, SP, CEP 13.100-268. E-mail: emiliac@fcm.unicamp.br

\section{Resumo}

Este estudo teve como objetivos conhecer a distribuição do peso de nascimento das crianças de Campinas (SP) e indicar fatores de risco para baixo peso. Realizou-se estudo transversal no qual foram utilizados dados das 14.444 Declarações de Nascidos Vivos de 2001. A variável dependente foi o peso de nascimento, e as independentes as características maternas, gestacionais, do parto e do recém-nascido. Na avaliação da associação entre variáveis empregou-se teste de qui-quadrado e calculou-se valores de odds ratio brutos (OR) e ajustados (ORaj). A média do peso de nascimento foi 3.142g, variando de 285 a $5.890 \mathrm{~g} ; 65,1 \%$ das crianças pesaram $3.000 \mathrm{~g}$ ou mais, $25,7 \%$ entre 2.500 e 2.999 g e $9,1 \%$ menos de 2.500 g. Os determinantes para baixo peso em prematuros foram cesariana, gemelaridade, recém-nascidos femininos e os de mulheres com menos de sete consultas de pré-natal. Para crianças a termo os riscos foram gestação dupla, tripla ou mais, filhos de mulheres com mais de 34 anos, das com até sete anos de estudo, com oito a onze e das com menos de sete consultas de pré-natal. A distribuição de peso de nascimento em Campinas foi inadequada e a proporção de baixo peso foi mais que o dobro dos países desenvolvidos. Os recém-nascidos prematuros que nasceram por cesariana, os prematuros e os a termo de gestação múltipla, os femininos, os de mulheres com pré-natal inadequado e os a termo daquelas com maior idade e baixa escolaridade apresentaram maior chance de nascer com baixo peso.

Palavras chave: Saúde materno-infantil. Peso ao nascer. Recém-nascido de baixo peso. Fatores de risco.

Aprovado pelo Comitê de Ética em Pesquisa da Faculdade de Ciências Médicas da UNICAMP, Parecer NNo. 362/2002 
Abstract

The distribution of birth weight was determined for children in Campinas, Brazil, and risk factors for low birth weight were assessed. A cross-sectional study was conducted using the variables from Live Birth Certificates in 2001. The dependent variable was birth weight and the independent ones were mothers' characteristics and those related to their pregnancies and to newborns. The association between variables was assessed by the chi-square test, crude oddsratio (OR) and adjusted odds-ratio (ORadj) values. The mean birth weight was $3,142 \mathrm{~g}$, with a distribution varying from 285 to $5,890 \mathrm{~g}$, in which $65.1 \%$ of the children weighed $3,000 \mathrm{~g}$ or more, $25.7 \%$ weighed from 2,500 to 2,999g and $9.1 \%$ presented low birth weight. The determinants for low birth weight for premature babies were cesarean sections, twin babies, female babies and mothers having less than seven medical visits in prenatal care. For term babies, the risk for low birth weight was increased for twins, triplets or more, mother more than 34 years old, those who studied up to seven years, or 8-11 years, and mothers having less than seven medical visits in prenatal care. The results indicate that the distribution of birth weight in Campinas was inadequate and the proportion of low birth weight was more than two times higher than in developed countries. The likelihood for low birth weight was higher for premature babies born by C-sections, preterm and term babies from a multiple pregnancy, female babies, babies of mothers with inappropriate prenatal care and term babies of older mothers and with a low educational level.

Keywords: Infant and maternal health. Birth weight. Low birth weight infant. Risk factors.

\section{Introdução}

O peso ao nascer é, provavelmente, $\mathrm{o}$ fator isolado mais importante relacionado à mortalidade neonatal, pós-neonatal e infantil, à morbidade na infância e ao risco de várias doenças na idade adulta ${ }^{1,2}$. Portanto, $o$ estudo da distribuição do peso de nascimento em uma determinada população fornece subsídios para que sejam implantadas ações que resultem em condições ideais de crescimento fetal, contribuindo para o desenvolvimento global do indivíduo.

As taxas de baixo peso ao nascer (BPN) são muito variáveis nas diversas regiões do mundo, com evidentes desvantagens para os países menos desenvolvidos, pois estão associadas a condições socioeconômicas desfavoráveis e podem ser consideradas como um indicador de nível de saúde da população. Assim, em países desenvolvidos da Europa, como Itália, França, Portugal, Suíça, Suécia, Noruega, Irlanda e Espanha, a porcentagem de BPN varia entre 4 a $5^{3}$. Nos Estados Unidos esta taxa é de 7,6\%, porém com variação de $13,1 \%$ na população negra e 4,8\% na branca ${ }^{4}$. As maiores proporções são as da Índia (33,0\%) e as de Bangladesh $(50,0 \%)^{3}$. Na América Latina e Caribe, o valor médio é de 9,0\%. No Brasil, em 2001, a média de BPN foi de 7,9\%, sendo a maior porcentagem no Estado do Rio de Janeiro $(9,4)$ e a menor em Roraima $(5,0)^{5}$. Em geral, nos países desenvolvidos estas taxas são principalmente decorrentes de partos prematuros, observados em dois terços dos nascimentos de crianças com BPN. Para os países em desenvolvimento, esta ocorrência se deve, na maioria das vezes, ao retardo de crescimento intra-uterino.

Entre os fatores associados ao BPN estão, entre outros, as infecções genitais, os partos múltiplos, a hipertensão arterial, as disfunções uterinas, o baixo índice de massa corporal materna pré-gestacional, a baixa estatura materna, o tabagismo na gravidez, a placenta prévia, o baixo ganho de peso na gestação, os fatores étnicos, a primiparidade, as anomalias congênitas e os fatores genéticos ${ }^{1}$. 
O presente estudo teve como objetivos conhecer a distribuição do peso de nascimento dos recém-nascidos de Campinas (SP), em 2001, e indicar fatores de risco para a ocorrência de BPN.

\section{Métodos}

Trata-se de um estudo transversal, realizado a partir de dados das 14.444 Declarações de Nascidos Vivos do Sistema de Informações sobre Nascidos Vivos (Sinasc), referentes aos partos de mulheres residentes em Campinas, ocorridos no próprio município em 2001.

A cidade de Campinas (SP), localizada a cerca de $100 \mathrm{~km}$ da capital paulista, é a terceira maior cidade do Estado, com uma população de 983.945 habitantes em 2001, sendo 98,4\% residentes em área urbana, tendo o oitavo Índice de Desenvolvimento Humano (IDH) do Estado. Com relação aos diferenciais nos níveis de qualidade de vida e de saúde da população, o indicador da Secretaria Municipal de Saúde - Índice de Condição de Vida (ICV) - apontou que a maioria das áreas de abrangência dos Centros de Saúde (CS) dos Distritos de Saúde (DS) Noroeste e Sudoeste pertencia ao grupo de pior ICV, e as do DS Leste ao de melhor ICV. As áreas dos CS nos DS Norte e Sul estiveram, mais homogeneamente, distribuídas entre o melhor, médio e pior $\mathrm{ICV}^{6}$.

As características das mães e dos RNs foram identificadas pelas variáveis sociodemográficas maternas (idade, região de moradia, escolaridade, ocupação, situação conjugal), as relacionadas à gestação (número de consultas de pré-natal, número de filhos vivos e mortos, tipo e duração da gestação), ao parto e ao RN (tipo de parto, peso e sexo do $\mathrm{RN}$ ).

Para a análise estatística, a variável dependente foi o peso de nascimento $(<2.500 \mathrm{~g}$ ou $\geq 2.500 \mathrm{~g}$ ). As variáveis independentes foram: situação conjugal (com companheiro e sem companheiro), DS de moradia (Norte, Sul, Leste, Noroeste e Sudoeste), escolaridade (até sete, de oito a 11 e de 12 e mais anos de estudo concluídos), trabalho fora do lar (sim e não), idade materna ( $<20$ anos, 20 a 34 anos e $\geq 35$ anos), duração da gestação em semanas (<37 ou $\geq 37$ ), número de filhos vivos tidos em gestações anteriores (zero, um, dois e $\geq$ três) e número de filhos mortos (zero, um e $\geq$ dois), número de consultas de pré-natal (<sete e $\geq$ sete), sexo do RN (masculino ou feminino), tipo de gestação (única, dupla e $\geq$ três), e tipo de parto (vaginal ou cesáreo).

Estudou-se a associação entre a variável dependente e as independentes utilizando-se o teste de qui-quadrado e valores de odds ratio brutos, calculados utilizando-se a rotina Statcalc do software EPIINFO versão 6.04 Empregou-se o modelo de regressão logística múltipla (método stepwise backward) usando o software SPPS 11.0, com o objetivo de obter os valores de odds ratio ajustados. Foram desenvolvidos dois modelos, sendo o primeiro para RNs pré-termo e o segundo para RNs a termo, e utilizou-se como critério de inclusão das variáveis $\mathrm{p}<0,20$ e de exclusão $\mathrm{p}>0,05$. Para averiguar diferença entre as médias de peso de nascimento foi utilizada análise de variância (ANOVA).

As categorias escolhidas como referência na análise univariada e multivariada foram as de menor risco esperado para o baixo peso de nascimento, conforme apontado pela literatura (DS Leste - o de melhor ICV, mãe com 20 a 34 anos de idade, com 12 anos ou mais de estudo concluídos, com trabalho fora do lar, com companheiro, sem filhos mortos, com um filho vivo anteriormente, com sete ou mais consultas de pré-natal, com gestação única, em gestação a termo, RN de sexo masculino e parto vaginal).

\section{Resultados}

O peso de nascimento variou de 285 a 5.890 g, com média de $3.142 \mathrm{~g}$, desvio-padrão de 539,9g e mediana de 3.175g. A maior média de peso foi a do DS Leste e a menor no DS Sul e Sudoeste e, apesar das diferenças nos ICV do município, não foi observada significância estatística entre as 
regiões (Tabela 1). A maioria das crianças $(65,1 \%)$ nasceu com peso igual ou superior a $3.000 \mathrm{~g}$ e $25,7 \%$ com peso entre 2.500 e 2.999g. A porcentagem de baixo peso ao nascer foi de 9,1\%. A distribuição em relação aos DS está apresentada na Tabela 2.

A distribuição de freqüência e os valores de OR brutos apresentados na Tabela 3 mostraram associação entre baixo peso de nascimento e os filhos de mulheres com idade igual ou maior que 35 anos, das moradoras do DS Sul, do Sudoeste, daquelas cuja escolaridade era até sete anos de estudo, ou de oito a 11 anos e que não trabalhavam fora do lar.

As crianças que nasceram prematuras, as do sexo feminino e as resultantes de gestações duplas ou triplas e mais também mostraram maiores riscos para baixo peso ao nascer. Notou-se, também, associação entre o BPN e os RN das mulheres sem filhos anteriormente, daquelas que já tinham dois filhos, três ou mais e das que fizeram menos que sete consultas de prénatal (Tabela 4).

Para a análise de regressão logística múltipla foram selecionadas as variáveis DS, tipo de gestação, idade, situação conjugal, trabalho fora do lar e escolaridade materna, número de consultas de pré-natal e sexo do RN.

Nos modelos para pré-termos e a termo houve associação entre o BPN e RNs nascidos de gestação múltipla, os do sexo

Tabela 1 - Distribuição do peso de nascimento (gramas) dos nascidos vivos segundo o Distrito de Saúde. Campinas, SP, 2001.

Table 1 - Distribution of newborn birth weight (grams) according to Health District. Campinas, SP, 2001.

\begin{tabular}{lccccc}
\hline Distrito de Saúde & Média $^{*}$ & Desvio Padrão & Mínimo & Mediana & Máximo \\
\hline Norte & 3.148 & 544,5 & 285 & 3.190 & 5.020 \\
Sul & 3.125 & 534,8 & 440 & 3.165 & 4.990 \\
Leste & 3.154 & 524,5 & 530 & 3.170 & 4.950 \\
Noroeste & 3.153 & 547,4 & 300 & 3.185 & 5.890 \\
Sudoeste & 3.131 & 546,9 & 560 & 3.168 & 5.200 \\
Campinas & 3.142 & 539,9 & 285 & 3.175 & 5.890 \\
\hline
\end{tabular}

* $\mathrm{p}=0,102$ (Análise de variância)

Excluído um nascimento por falta de registro de peso de nascimento e um sem registro de local de moradia

Excluded one newborn without birth weight record and one without record of place of residence

Tabela 2 - Distribuição dos nascidos vivos segundo o peso de nascimento (gramas) e o Distrito de Saúde. Campinas, SP, 2001.

Table 2 - Distribution of newborns according to birth weight (grams) and Health District. Campinas, SP, 2001.

\begin{tabular}{|c|c|c|c|c|c|c|}
\hline \multirow{3}{*}{$\begin{array}{l}\text { Distrito de } \\
\text { Saúde }\end{array}$} & \multicolumn{6}{|c|}{ Peso de nascimento (gramas) ${ }^{*}$} \\
\hline & \multicolumn{2}{|c|}{$<2.500$} & \multicolumn{2}{|c|}{$2.500 “ \% 3000$} & \multicolumn{2}{|c|}{$\geq 3000$} \\
\hline & $\mathrm{N}$ & $(\%)$ & $\mathrm{N}$ & $(\%)$ & $\mathrm{N}$ & $(\%)$ \\
\hline Norte & 216 & 9,1 & 593 & 25,1 & 1.556 & 65,8 \\
\hline Sul & 353 & 9,6 & 942 & 25,6 & 2.388 & 64,8 \\
\hline Leste & 194 & 7,8 & 655 & 26,1 & 1.658 & 66,1 \\
\hline Noroeste & 255 & 10,0 & 671 & 26,3 & 1.628 & 63,7 \\
\hline Sudoeste & 297 & 9,0 & 858 & 25,7 & 2.178 & 65,3 \\
\hline Campinas & 1.315 & 9,1 & 3.719 & 25,7 & 9.408 & 65,1 \\
\hline
\end{tabular}

* $p=0,223$ (Teste do qui-quadrado)

Excluído um nascimento por falta de registro de peso ao nascer e um sem registro de local de moradia

$\mathrm{N}$ : freqüência absoluta \%: freqüência relativa

Excluded one newborn without birth weight record and one without record of place of residence

$\mathrm{N}$ : absolute frequency \%: relative frequency 
Tabela 3 - Distribuição de freqüência e valores de odds ratio brutos do peso de nascimento em relação à situação conjugal, ao Distrito de Saúde, à escolaridade, à ocupação e à idade materna. Campinas, SP, 2001.

Table 3 - Frequency and crude odds ratio values for birth weight according to marital status, Health District, maternal schooling, maternal job and maternal age. Campinas, SP, 2001.

\begin{tabular}{|c|c|c|c|c|c|c|}
\hline \multirow[t]{2}{*}{ Categorias de análise } & \multirow[t]{2}{*}{$\mathrm{N}$} & \multicolumn{2}{|c|}{$<2.500 \mathrm{~g}$} & \multirow[t]{2}{*}{$\mathrm{OR}^{1}$} & \multirow[t]{2}{*}{ IC95\% ${ }^{1}$} & \multirow[t]{2}{*}{$\mathrm{p}^{1}$} \\
\hline & & $\mathrm{N}$ & $\%$ & & & \\
\hline \multicolumn{7}{|l|}{ Situação conjugal } \\
\hline Sem companheiro & 5.165 & 498 & 9,6 & 1,11 & $0,98-1,25$ & 0,090 \\
\hline Com companheiro & 9.242 & 813 & 8,8 & 1,00 & & \\
\hline \multicolumn{7}{|l|}{ Distrito de Saúde } \\
\hline Norte & 2.365 & 216 & 9,1 & 1,20 & $0,97-1,47$ & 0,079 \\
\hline Sul & 3.683 & 353 & 9,6 & 1,26 & $1,05-1,52$ & 0,011 \\
\hline Leste & 2.507 & 194 & 7,7 & 1,00 & & \\
\hline Noroeste & 3.333 & 297 & 8,9 & 1,17 & $0,96-1,42$ & 0,109 \\
\hline Sudoeste & 2.554 & 255 & 10,0 & 1,32 & $1,08-1,62$ & 0,004 \\
\hline \multicolumn{7}{|l|}{ Escolaridade da mãe } \\
\hline Até 7 & 5.389 & 541 & 10,0 & 1,52 & $1,25-1,84$ & $<0,001$ \\
\hline 8 a 11 & 6.722 & 611 & 9,1 & 1,36 & $1,12-1,64$ & 0,001 \\
\hline 12 e mais & 2.158 & 148 & 6,8 & 1,00 & & \\
\hline \multicolumn{7}{|l|}{ Trabalho fora do lar } \\
\hline Não & 8.736 & 850 & 9,7 & 1,21 & $1,07-1,36$ & 0,001 \\
\hline Sim & 5.688 & 466 & 8,2 & 1,00 & & \\
\hline \multicolumn{7}{|l|}{ Idade materna (anos) } \\
\hline$<20$ & 2.562 & 248 & 9,7 & 1,12 & $0,97-1,30$ & 0,127 \\
\hline 20 “\% 35 & 10.366 & 904 & 8,7 & 1,00 & & \\
\hline$\geq 35$ & 1512 & 164 & 10,8 & 1,27 & $1,06-1,52$ & 0,006 \\
\hline
\end{tabular}

N: freqüência absoluta \%: freqüência relativa OR: Odds ratio IC95\%: Intervalo de confiança de 95\% p: probabilidade do teste do qui-quadrado $\mathrm{N}$ : absolute numbers \%: relative frequency OR: Odds ratio $95 \%$ IC: $95 \%$ Confidence Interval for $\mathrm{p}$ : chi-square probability test

feminino e os filhos de mulheres que fizeram menos que sete consultas de pré-natal. As mulheres com mais de 34 anos de idade, as com até sete anos de estudo, as com oito a onze anos e que tiveram parto a termo apresentaram risco aumentado de terem filhos com menos de $2.500 \mathrm{~g}$. O parto cesáreo mostrou associação com BPN das crianças prematuras (Tabela 5).

\section{Discussão}

Neste estudo verificou-se que $65,1 \%$ das crianças nasceram com $3.000 \mathrm{~g}$ ou mais, considerado como peso adequado, sendo a média $3.142 \mathrm{~g}$, com pequenas variações em relação aos DS. Esta situação de distribuição de peso em Campinas pouco se modificou em relação a 1995, quando foram encontradas proporções e médias de peso bastante semelhantes ${ }^{7}$. Em outras localidades brasileiras foram verificadas médias diferentes das do município, como em São Paulo em 1998 (3.157g) ${ }^{8}$, em Ribeirão Preto (SP) em $1994(3.115 \mathrm{~g})^{9}$, em São Luís (MA) em 1997 e $1998(3.142 \mathrm{~g})^{10}$, e no Estado de Goiás, onde foram avaliados somente os nascimentos provenientes de gestações únicas ${ }^{11}$.

Esta distribuição de peso ao nascimento não é a ideal, pois, segundo recomendação da OMS, a proporção de nascidos com peso adequado deve ser de pelo menos $85 \%$ e o peso médio ao nascimento deve estar entre $3.400 \mathrm{~g}$ e $3.500 \mathrm{~g}^{8,12}$.

O BPN, que pode ser decorrente de prematuridade ou de retardo de crescimento intra-uterino, tem sido objeto de vários estudos em virtude de participação importante na mortalidade e morbidade infantis e riscos para a saúde na vida adulta. As crianças nascidas com menos de $2.500 \mathrm{~g}$ têm ris- 
Tabela 4 - Distribuição de freqüência e valores de odds ratio brutos do peso de nascimento em relação à duração e tipo da gestação, ao número de filhos nascidos vivos e mortos, ao número de consultas de pré-natal e ao sexo do recémnascido. Campinas, SP, 2001.

Table 4 -Frequency and crude odds ratio values for birth weight according to duration and type of gestation, number of children alive and dead, number of prenatal visits, and newborn gender. Campinas, SP, 2001.

\begin{tabular}{|c|c|c|c|c|c|c|}
\hline \multirow[t]{2}{*}{ Categorias de análise } & \multirow[t]{2}{*}{$\mathrm{N}$} & \multicolumn{2}{|c|}{$<2.500 \mathrm{~g}$} & \multirow[t]{2}{*}{$\mathrm{OR}^{1}$} & \multirow[t]{2}{*}{ IC $95 \%{ }^{1}$} & \multirow[t]{2}{*}{$p^{1}$} \\
\hline & & $\mathrm{N}$ & $\%$ & & & \\
\hline \multicolumn{7}{|l|}{ Duração da gestação } \\
\hline$<37$ & 1.017 & 647 & 63,6 & 34,74 & $29,80-40,50$ & $<0,01$ \\
\hline$\geq 37$ & 13.313 & 638 & 4,8 & 1,00 & & \\
\hline \multicolumn{7}{|l|}{ Filhos mortos } \\
\hline 0 & 13.243 & 1.194 & 9,0 & 1,00 & & \\
\hline 1 & 971 & 97 & 10,0 & 1,12 & $0,89-1,40$ & 0,308 \\
\hline$\geq 2$ & 214 & 25 & 11,6 & 1,33 & $0,86-2,07$ & 0,177 \\
\hline \multicolumn{7}{|c|}{ Filhos vivos em gestações anteriores } \\
\hline 0 & 6583 & 636 & 9,6 & 1,35 & $1,17-1,55$ & $<0,001$ \\
\hline 1 & 4.417 & 325 & 7,3 & 1,00 & & \\
\hline 2 & 1.939 & 190 & 9,8 & 1,37 & $1,13-1,66$ & 0,001 \\
\hline$\geq 3$ & 1.495 & 165 & 11,0 & 1,56 & $1,28-1,91$ & $<0,001$ \\
\hline \multicolumn{7}{|l|}{ Consultas de pré-natal } \\
\hline$<7$ & 3.354 & 565 & 16,9 & 3,22 & $2,84-3,65$ & $<0,001$ \\
\hline$\geq 7$ & 9.733 & 576 & 5,9 & 1,00 & & \\
\hline \multicolumn{7}{|l|}{ Sexo } \\
\hline Masculino & 7332 & 599 & 8,2 & 1,00 & & \\
\hline Feminino & 7108 & 715 & 10,0 & 1,26 & $1,12-1,41$ & $<0,001$ \\
\hline \multicolumn{7}{|l|}{ Tipo de gestação } \\
\hline Única & 14.171 & 1.140 & 8,0 & 1,00 & & \\
\hline Dupla & 255 & 168 & 65,9 & 22,07 & $16,78-29,05$ & $<0,001$ \\
\hline$\geq 3$ & 16 & 08 & 50,0 & 11,43 & $3,91-33,45$ & $<0,001$ \\
\hline \multicolumn{7}{|l|}{ Tipo de parto } \\
\hline Vaginal & 6.514 & 565 & 8,7 & 1,00 & & \\
\hline Cesáreo & 7.925 & 751 & 9,5 & 1,10 & $0,98-1,24$ & 0,095 \\
\hline
\end{tabular}

N: freqüência absoluta \%: freqüência relativa OR: Odds ratio IC95\%: Intervalo de confiança de 95\% p: probabilidade do teste do qui-quadrado

$\mathrm{N}$ : absolute numbers \%: relative frequency OR: Odds ratio 95\% IC: $95 \%$ Confidence Interval for p: chi-square probability test

co aumentado de morte no primeiro ano de vida, de desenvolverem enfermidades infecciosas e respiratórias e de terem atraso de crescimento e desenvolvimento $^{1,2}$. Além disso, estudos sugerem que estas crianças podem, no futuro, apresentar doenças cardíacas, acidente vascular cerebral, hipertensão arterial, diabetes tipo 2, hiperlipidemia e obesidade ${ }^{13-15}$.

A taxa de BPN encontrada nesse estudo foi $9,1 \%$, bastante semelhante à do Estado de São Paulo e pouco acima da região de Campinas $(8,9 \% \text { e } 8,5 \% \text {, respectivamente })^{5}$, mas mais que o dobro da encontrada para os países desenvolvidos ${ }^{3,4}$. Dessas crianças, quase metade nasceu com 37 semanas ou mais de gestação, sugerindo que a cidade de Campinas, apesar de ter um dos melhores IDH do país, encontra-se em uma situação intermediária entre a dos países desenvolvidos, que têm o maior percentual de $\mathrm{BPN}$ devido à prematuridade, e a dos em desenvolvimento, com maior ocorrência devido ao retardo de crescimento intrauterino.

A incidência de BPN, que tem se mantido constante nas três últimas décadas ${ }^{7}$, concorda com os valores encontrados para região, Estado e outros municípios ${ }^{5}$ e com a média da América Latina ${ }^{3}$. Tendência semelhante foi encontrada em São Paulo; entretanto quando a análise foi feita por 
Tabela 5 - Valores de odds-ratio ajustados do peso de nascimento de recém-nascidos prétermo e a termo associados a variáveis sócio-demográficas maternas, gestacionais e às relacionadas ao parto e às condições do nascimento. Campinas, SP, 2001.

Table 5 -Adjusted odds ratio for birth weight of preterm and term newborns associated with mother socio-demographic, pregnancy, delivery, and birth condition variables. Campinas, SP, 2001.

\begin{tabular}{lccc}
\hline Variável & OR ajustada & IC 95\% & $\mathrm{p}$ \\
\hline RN PRÉ-TERMO & & & \\
Sexo feminino & 1,99 & $1,47-2,70$ & $<0,001$ \\
$<7$ consultas pré-natal & 3,59 & $2,61-4,92$ & $<0,001$ \\
Gestação dupla & 14,82 & $5,32-41,27$ & $<0,001$ \\
Parto Cesáreo & 1,52 & $1,10-2,10$ & 0,0112 \\
RN TERMO & & & \\
Sexo feminino & 1,53 & $1,28-1,83$ & $<0,001$ \\
$<7$ consultas pré-natal & 1,88 & $1,56-2,28$ & $<0,001$ \\
Gestação dupla & 19,87 & $13,57-29,09$ & $<0,001$ \\
Gestação tripla & 21,40 & $5,68-80,65$ & $<0,001$ \\
<7 anos de escolaridade & 1,74 & $1,23-2,48$ & 0,0018 \\
8 a 11anos de escolaridade & 1,63 & $1,18-2,27$ & 0,0032 \\
Idade materna $\geq 35$ anos & 1,41 & $1,05-1,88$ & 0,0192 \\
\hline
\end{tabular}

OR: Odds ratio IC 95\%: Intervalo de confiança de $95 \%$

OR: Odds ratio IC 95\%: 95\% confidence interval

estratos sociais, verificaram-se trajetórias favoráveis do peso ao nascer nos estratos de menor nível socioeconômico, aparentemente devido à melhoria das condições e hábitos de vida e de acesso a serviços de saúde, e desfavoráveis nos de maior nível, pelo aumento da freqüência de RN prematuros $^{8}$. Em Ribeirão Preto (SP), apesar do declínio da mortalidade infantil, houve aumento das taxas de BPN num intervalo de 15 anos, principalmente nos estratos sociais de maior renda, associado provavelmente ao aumento de cesarianas ${ }^{16}$. Em Pelotas (RS), embora tenha havido um aumento nas taxas de BPN de 1982 a 1993, assim como em São Paulo, houve declínio da prevalência de BPN nas famílias de muito baixa renda ${ }^{17}$.

Portanto, pode-se supor que há diversos fatores associados ao BPN e, conforme apontado por Silva et al. ${ }^{18}$, sua ocorrência não deve ser sistematicamente considerada como um indicador de desenvolvimento social

O BPN foi fortemente influenciado pela prematuridade, ou seja, as crianças nascidas antes de 37 semanas tiveram quase 35 vezes mais chance de pesar menos de
$2.500 \mathrm{~g}$ do que as de maior idade gestacional. Em São Paulo também se encontrou que os $\mathrm{RN}$ nascidos prematuros apresentaram um risco mais de 20 vezes maior que o observado para os nascidos a termo, em todos os estratos de escolaridade ${ }^{8}$. Aragão et al. ${ }^{19}$ verificaram que os fatores de risco para prétermos no Maranhão localizaram-se nas mulheres menores de 18 anos e naquelas que não freqüentaram o programa de prénatal. Estudo desenvolvido em Goiás encontrou, além desses, risco para mulheres com mais de 35 anos e para moradoras em cidades do interior do Estado ${ }^{11}$.

Foi encontrada associação entre BPN dos RN nascidos pré-termo e parto cesáreo. A ocorrência de partos prematuros pode estar associada a diversos fatores, como os socioeconômicos, obstétricos, ginecológicos, clínico-cirúrgicos, iatrogênicos e os de etiologia desconhecida, sendo que o parto cirúrgico pode ser indicado em algumas dessas situações. Por não ser objetivo desse estudo, não foi possível avaliar estas indicações, sendo necessárias pesquisas que abordem este tema especificamente.

No entanto, vale ressaltar que a ocorrência de partos prematuros apresentou 
tendência ascendente em Campinas (5,9\% em 1.997 e 7,0\% em 2001), observado também por Mariotoni e Barros-Filho ${ }^{12}$, no período de 1971 a 1995, em uma maternidade do município, que supuseram ser este fato decorrente do aumento das gestações na adolescência e da interrupção precoce da gravidez por cesárea. Do mesmo modo, estudos realizados em Ribeirão Preto e em Pelotas sugeriram que as altas taxas de partos cesáreos foram parcialmente responsáveis pelo aumento da prematuridade e da conseqüente diminuição na média do peso de nascimento ${ }^{16,20}$.

Nesse estudo, verificou-se que as mães com menor escolaridade (menos de sete anos de estudo e de oito até onze anos) tiveram chance 1,7 vezes maior de terem filhos a termo com BPN do que aquelas com mais tempo de estudo. Haidar et al. ${ }^{21}$, estudando a correlação entre escolaridade materna com indicadores obstétricos, obtiveram resultados semelhantes e concluíram que tal associação pode estar relacionada à pior condição socioeconômica das mães, que pode afetar o ganho de peso durante a gestação e a inadequada freqüência ao pré-natal. Monteiro et al. ${ }^{8}$, estudando a tendência secular do peso ao nascer na cidade de São Paulo (SP), verificaram que a sua distribuição pouco se modificou em 15 anos e que, embora modestamente, o risco para BPN aumentou à medida que diminuía a escolaridade das gestantes.

Considerando, então, a escolaridade como marcador da condição socioeconômica da mãe e de sua família, visto estar altamente relacionada à ocupação e à ren$\mathrm{da}^{1}$, os achados deste estudo concordam com os de Horta et al. ${ }^{17}$, que observaram, em Pelotas (RS), em 1982 e 1993, que a renda familiar esteve inversamente associada com o BPN e o retardo de crescimento intrauterino, e com os de Zambonato et al., que verificaram que as crianças nascidas em família cuja renda era menor que um salário mínimo tinham maior risco de $\mathrm{BPN}^{22}$.

As mulheres com idade superior a 34 anos tiveram 1,4 vezes mais chance de te- rem filhos pequenos para a idade gestacional, resultado coerente com o encontrado na literatura ${ }^{11,16,23}$ e parece estar relacionado com maior paridade, doenças maternas pré-existentes e maior incidência de intercorrências na gravidez.

Observou-se também a associação entre BPN e crianças do sexo feminino nascidas prematuras ou a termo, como descrito em estudos anteriores ${ }^{11,12,16,23,24}$. Embora Kramer ${ }^{1}$ tenha verificado que para as crianças com baixo peso devido à prematuridade, não haja diferença entre os sexos, os meninos apresentam maior peso ao nascer e menor risco de retardo de crescimento intra-uterino, tanto em países desenvolvidos quanto naqueles em desenvolvimento. Apesar dessa constatação e dos achados nos diversos estudos, o sexo do RN é uma variável em que não há possibilidade de intervenção.

As crianças nascidas de partos de duplos e de triplos tiveram maior chance de nascerem com peso inferior a $2.500 \mathrm{~g}$, dado provavelmente decorrente do fato que as gestações múltiplas predispõem ao trabalho de parto prematuro. Beiguelman et al. ${ }^{25}$, estudando $\mathrm{RN}$ de partos gemelares em três cidades do sudeste brasileiro, verificaram que o padrão da taxa de crescimento fetal dos gêmeos é retardado, quando comparados aos $\mathrm{RN}$ de partos únicos, independente da classe social e econômica das mães, e que o crescimento dos fetos de sexo feminino foi levemente inferior aos masculinos depois de 28 semanas de gestação.

Em relação ao pré-natal, as mulheres que fizeram menos de sete consultas tiveram mais chance de ter filhos com BPN do que as que freqüentaram o programa adequadamente. Este achado concordou com os de pesquisa realizada no município do Rio de Janeiro (RJ), na qual se observou que a atenção pré-natal contribuiu favoravelmente para o peso de nascimento, mesmo quando controlados outros fatores, tais como situação conjugal, hábito de fumar, história reprodutiva, ocorrência de diabetes e prematuridade na atual gestação ${ }^{26}$. No mes- 
mo sentido, Kilsztajn et al. ${ }^{27}$, estudando a influência do pré-natal sobre a ocorrência de BPN e prematuridade, verificaram que o número de consultas está inversamente relacionado à prevalência desses desfechos. Em estudo realizado na cidade de São Paulo (SP), constatou-se também que as mães que fizeram menos que cinco consultas durante a gestação, apresentaram um risco relativo duas vezes e meia maior de BPN do que aquelas que retornaram ao pré-natal cinco vezes ou mais ${ }^{8}$. Em Pelotas (RS), Victora e Barros $^{28}$ apontaram que a assistência prénatal é potencialmente uma medida de alto impacto na redução da mortalidade infantil devido a causas perinatais, e Zambonato et al. ${ }^{22}$ observaram associação entre a baixa qualidade do pré-natal e o nascimento de crianças pequenas para a idade gestacional, sugerindo que além do número adequado de consultas, deve-se também buscar uma melhora na qualidade da atenção. .

No entanto, têm ocorrido questionamentos sobre o impacto do pré-natal para prevenir o BPN. Estudo realizado por Hueston et al. ${ }^{29}$ mostrou que não houve redução das taxas de BPN para os filhos de mulheres norte-americanas que iniciaram o pré-natal precocemente. Os fatores que se relacionaram ao $\mathrm{BPN}$ entre as que iniciaram o cuidado tardiamente em relação às que o fizeram precocemente foram provavelmente relacionados às características sociodemográficas maternas. Neste sentido, Kramer ${ }^{1}$ aponta que as mulheres que iniciam o pré-natal tardiamente e, conseqüentemente, comparecem a um menor número de consultas, comumente são adolescentes, primíparas, de pior nível socioeconômico, malnutridas e provavelmente usuárias de fumo, álcool ou outras drogas, fatores que contribuem para desfechos desfavoráveis para o $\mathrm{RN}$, entre eles o baixo peso.

Silveira e $\operatorname{Santos}^{30}$, em revisão sistemática da literatura de publicações sobre a influência do pré-natal sobre o BPN, encontraram resultados conflitantes entre os estudos. Heterogeneidade de indicadores, vieses de auto-seleção, dados secundários com baixa acurácia ou informações ignoradas, fatores individuais maternos e de organização de serviços, entre outros, foram apontados como possíveis confundidores, podendo influir nos resultados encontrados e limitar a qualidade dos estudos.

Contudo, a preocupação com a adequada assistência pré-natal não deve se limitar à prevenção do BPN, visto que, por meio deste acompanhamento pode-se orientar as gestantes quanto ao cuidado com a própria saúde e adoção de hábitos de vida saudáveis para si e para seu filho, identificar situações de riscos para a gestação e o parto, aplicar intervenções oportunas e evitar desfechos desfavoráveis para a mãe ou para o bebê, além da possibilidade de mantê-la vinculada ao programa de atenção à saúde da mulher e da criança para seguimentos posteriores.

Assim, estudos mais amplos e com diferentes abordagens são necessários para o esclarecimento dos reais benefícios do pré-natal na gestação, no parto e no puerpério, e para nortear programas de assistência que realmente contribuam para a saúde das mães e das crianças.

Os resultados desse estudo mostraram que o município, apesar de ter um dos melhores Índices de Desenvolvimento Humano do país, não apresentou média de peso ao nascer e taxa de BPN compatíveis para localidades desenvolvidas.

A análise dos fatores de risco sugere que os recém-nascidos prematuros que nasceram por cesariana, os prematuros e os a termo de gestação múltipla, os femininos, os de mulheres com pré-natal inadequado e os a termo das maiores de 34 anos e daquelas com baixa escolaridade apresentaram maior chance de nascer com baixo peso.

A limitação desse estudo foi a impossibilidade de avaliar todas as situações que poderiam determinar o BPN, visto que a análise baseou-se somente nas variáveis das Declarações de Nascidos Vivos do Sinasc. No entanto, os resultados encontrados nortearão novos estudos e poderão contribuir para a implementação de polí- 
ticas de saúde materno-infantil. A identificação precoce de fatores de risco para o nascimento de crianças com baixo peso por meio de instrumentos do cotidiano pode indicar estratégias preventivas rápidas e eficazes.

\section{Referências}

1. Kramer MS. Determinants of low birth weight: methodological assessment and meta-analysis. Bull World Health Organ 1987; 65(5): 663-737.

2. Wilcox AJ. On the importance - and the unimportanceof birth weight. Int J Epidemiol 2001; 30(6): 1233-41.

3. UNICEF. The state of the world's children 2000. Disponível em hhtp:/ / www.unicef.org. [Acessado em 22 de março de 2004].

4. Fang J, Madhavan S, Alderman MH. Low birth weight: race and maternal nativity - impact of community income. Pediatrics 1999; 103(1): [Inserir número de página]. Disponível em http://www.pediatrics.org/cgi/ content/full/103/1/e5 [Acessado em 27 de abril de 2005].

5. BRASIL. Ministério da Saúde. Sistema de Informações de Nascidos Vivos (Sinasc). Disponível em http:// www.funasa.gov.br/sis/sis02htm.2003. [Acessado em 12 de maio de 2004].

6. CAMPINAS. Secretaria Municipal de Saúde. Disponível em http://www.campinas.sp.gov.br/saude. [Acessado em 5 de outubro de 2004].

7. Bicalho-Mariotoni GG, Barros Filho AA. Nascer em Campinas: análise de dados do Sinasc, 1995. Rev Paul Pediatria 1997; 15(1): 24-30.

8. Monteiro CA, Benício MHD, Ortiz, LP. Tendência secular do peso ao nascer na cidade de São Paulo (1976 - 1998). Rev Saúde Pública 2000; 34(6 Supl): 26-40.

9. Barbieri MA, Silva AAM, Bettiol H, Gomes UA. Risk factors for the increasing trend in low birth weight among live births born by vaginal delivery, Brazil. Rev Saúde Pública 2000; 34(6): 596-02.

10. Silva AAM, Coimbra LC, Silva RA, Alves MTSSB, LamyFilho F, Lamy ZC et al. Perinatal health and mother-child health care in the municipality of São Luís, Maranhão State, Brazil. Cad Saúde Pública 2001; 17(6): 1413-26.

11. Faria RM. Fatores de risco para nascidos vivos de baixo peso, pré-termos e pequenos para a idade gestacional em Goiás [dissertação de mestrado]. Goiânia: Rede CentroOeste, UnB-UFG-UFMS; 2004

12. Mariotoni GGB, Barros Filho AA. A gravidez na adolescência é fator de risco para baixo peso ao nascer? $J$ Pediatr 1998; 74(2): 107-13.

13. Puffer RR, Serrano CV. Caracteristicas del peso al nascer. Washington, USA: PAHO; 1987 (Publicación Científica ${ }^{\circ}$ 504).
14. Law CM. Significance of birth weight for the future. Arch Dis Child Fetal Neonatal Ed 2002; 86: 7-8.

15. Ricciardi C, Guastadisegni C. Environmental inequities and low birth weight. Ann Ist Super Sanità 2003; 39(2): 229-34.

16. Silva AAM, Barbieri MA, Gomes UA, Bettiol H. Trends in low birth weight: a comparison of two birth cohorts separated by a 15-year interval in Ribeirão Preto, Brazil. Bull World Health Organ 1998; 76(1): 73-84.

17. Horta BL, Barros FC, Halpern R, Victora CG. Baixo peso ao nascer em duas coortes de base populacional no sul do Brasil. Cad Saúde Pública 1996; 12.(Supl.1): 27-31.

18. Silva AAM, Bettiol H, Barbieri MA, Ribeiro VS, Aragão VMF, Brito LGO, Pereira MM. Infant mortality and low birth weight in cities of Northeastern and Southeastern Brazil. Rev Saúde Pública 2003; 37(6): 693-98.

19. Aragão VMF, Silva AAM, Aragão LF, Barbieri MA, Bettiol H, Coimbra LC et al. Risk factors for preterm births in São Luis, Maranhão, Brazil. Cad Saúde Pública 2004; 20(1): 57-63.

20. Barros FC, Victora CG, Barros AJD, Santos IS, Albernaz E, Matijasevich A et al. The challenge of reducing neonatal mortality in middle-income countries: findings from three Brazilian birth cohorts in 1982, 1993 and 2004. Lancet 2005; 365(9462): 847-54.

21. Haidar FH, Oliveira UF, Nascimento LFC. Escolaridade materna: correlação com os indicadores obstétricos. Cad Saúde Pública 2001; 17(4): 1025-29.

22. Zambonato AMK, Pinheiro RT, Horta BL, Tomasi E. Fatores de risco para nascimento de crianças pequenas para idade gestacional. Rev Saúde Pública 2004; 38(1):24-9.

23. Costa CE, Gotlieb SL. Estudo epidemiológico do peso ao nascer a partir da Declaração de Nascido Vivo. Rev Saúde Pública 1998; 32(4): 328-34.

24. Barros FC, Victora CG, Vahghan P, Estanislau HJ. Bajo peso al nacer en el municipio de Pelotas, Brasil: Factores de riesgo. Bol of Sanit Panam 1987; 102(6): 541-54.

25. Beiguelman B, Colletto GDD, Franchi-Pinto C, Krieger H. Birth weight of twins: 1 . The fetal growth patterns of twins and singletons. Genet Mol Biol 1998; 21(1); [Inserir números de páginas]. Disponível em http:// www.scielo.br. [Acessado em 13 de fevereiro de 2005].

25. Leal MC, Gama SGN, Ratto KMN, Cunha CB. Uso do índice de Kotelchuck modificado na avaliação da assistência pré-natal e sua relação com as características maternas e o peso do recém-nascido no município do Rio de Janeiro. Cad Saúde Pública 2004; 20(Supl1): S63-S72. 
26. Kilsztajn S, Rossbach A, Carmo MSN, Sugahara TL. Assistência pré-natal, baixo peso e prematuridade no estado de São Paulo, 2000. Rev Saúde Pública 2003; 37(3): 303-10.

27. Victora CG, Barros FC. Infant mortality due to perinatal causes in Brazil: trends, regional patterns and possible interventions. Rev Paul Med 2001; 119(1): 33-42.
28. Hueston JH, Gregory, EG, Davis, L, Sturgill, V. Delayed prenatal care and the risk of low birth weight delivery. $J$ Community Health 2003; 28(\#):199-208.

29. Silveira DS, Santos IS. Adequação do pré-natal e peso ao nascer: uma revisão sistemática. Cad Saúde Pública 2004; 20(5): 1160-68.

Recebido em: 11/01/07 Versão final reapresentada em: 02/10/07 Aprovado em: 11/12/07 